\title{
Modeling the Specific Surface Area of Doped Spinel Ferrite Nanomaterials Using Hybrid Intelligent Computational Method
}

\author{
Taoreed O. Owolabi $\mathbb{D}^{1}{ }^{1}$ Tawfik A. Saleh, ${ }^{2}$ Olubosede Olusayo, ${ }^{3}$ Miloud Souiyah, ${ }^{4}$ \\ and Oluwatoba Emmanuel Oyeneyin ${ }^{5}$ \\ ${ }^{1}$ Physics and Electronics Department, Adekunle Ajasin University, Akungba Akoko, 342111 Ondo, Nigeria \\ ${ }^{2}$ Chemistry Department, King Fahd University of Petroleum and Minerals, Dhahran 31261, Saudi Arabia \\ ${ }^{3}$ Physics Department, Federal University Oye Ekiti, Oye, Ekiti State, Nigeria \\ ${ }^{4}$ Department of Mechanical Engineering, College of Engineering, University of Hafr Al Batin, P.O. Box 1803, \\ Hafr Al Batin 31991, Saudi Arabia \\ ${ }^{5}$ Theoretical and Computational Chemistry Unit, Department of Chemical Sciences, Adekunle Ajasin University, Akungba-Akoko, \\ Ondo State, Nigeria
}

Correspondence should be addressed to Taoreed O. Owolabi; owolabitaoreedolakunle@gmail.com

Received 30 May 2021; Revised 6 August 2021; Accepted 8 August 2021; Published 20 August 2021

Academic Editor: P. Davide Cozzoli

Copyright (C) 2021 Taoreed O. Owolabi et al. This is an open access article distributed under the Creative Commons Attribution License, which permits unrestricted use, distribution, and reproduction in any medium, provided the original work is properly cited.

\begin{abstract}
Spinel ferrites nanomaterials are magnetic semiconductors with excellent chemical, magnetic, electrical, and optical properties which have rendered the materials useful in many technological driven applications such as solar hydrogen production, data storage, magnetic sensing, converters, inductors, spintronics, and catalysts. The surface area of these nanomaterials contributes significantly to their targeted applications as well as the observed physical and chemical features. Experimental doping has shown a great potential in enhancing and tuning the specific surface area of spinel ferrite nanomaterials while the attributed experimental challenges call for viable theoretical model that can estimate the surface area of doped spinel ferrite nanomaterials with high degree of precision. This work develops stepwise regression (STWR) and hybrid genetic algorithmbased support vector regression (GBSVR) intelligent model for estimating specific surface area of doped spinel ferrite nanomaterials using lattice parameter and the size of nanoparticle as descriptors to the models. The developed hybrid GBSVR model performs better than STWR model with the performance improvement of $7.51 \%$ and $22.68 \%$, respectively, using correlation coefficient and root mean square error as performance metrics when validated with experimentally measured specific surface area of doped spinel ferrite nanomaterials. The developed GBSVR model investigates the influence of nickel, yttrium, and lanthanum nanoparticles on the specific surface area of different classes of spinel ferrite nanomaterials, and the obtained results agree excellently well with the measured values. The accuracy and precision characterizing the developed model would be of immense importance in enhancing specific surface area of doped spinel ferrite nanomaterial prediction with circumvention of experimental stress coupled with reduced cost.
\end{abstract}

\section{Introduction}

Spinel ferrite nanomaterials have gained a significant and considerable attention lately due to their unique chemical, physical, magnetic, electrical, and optical features that are of great interest in many technological advancement and applications such as gas sensor, drug-delivery, photocatalysts, water splitting, spintronics, and supercapacitors [1-4]. Other important characteristics of spinel ferrite nano- materials that promote their wider applicability include stability, being less expensive, and ease of preparation [5]. The specific surface area of spinel ferrite nanomaterials contributes significantly to their technological applications especially during organic pollutant treatment [6-8]. The significance of specific surface area and other physical properties of catalysts to pollutant treatment has been treated elsewhere [9-11]. Tuning of specific surface area of spinel ferrite nanomaterials is often carried out experimentally 
through doping whereby foreign and external materials are incorporated into the parent spinel ferrite ceramic compounds and consequently leads to alteration in magnetic, electrical, and optical properties coupled with change in specific surface area of the nanomaterials [12-15]. This work models the specific surface area of spinel ferrite nanomaterials doped with foreign materials through stepwise regression-based model and hybrid genetic algorithmbased support vector regression (GBSVR) intelligent computational method using lattice parameter and the size of the nanomaterial as descriptors to the models.

Using crystal structure as a yardstick of classification, ferrite family can be hexagonal, garnets, or spinel ferrite [6]. The unique properties of spinel ferrites among other ferrite family distinguish them from others and have offered them a place in several technological applications. Interstitial sites in spinel ferrite over which cations are distributed include octahedral and tetrahedral sites [16-18]. Variation of charge distribution in these sites alters the specific surface area as well as other physical and chemical properties of the nanomaterials while the introduction of external materials in the crystal structure redistributes charges within the available sites $[19,20]$. Doping that accompanies specific surface area and other physical properties enhancement distorts the lattice structure of the parent spinel which makes lattice distortion becomes significant while modeling the influence of dopants incorporation on the specific surface area of the nanomaterials. Inclusion of the size of nanomaterial among the descriptors is of importance since the exhibited interesting physical and chemical properties of spinel ferrite nanomaterials are constrained to the nanoscale size of the materials and display distinct features when investigated outside nanoscale [7]. A hybrid of genetic and support vector regression algorithms presented in this work exclusively models the nonlinear relationship between the specific surface area and the descriptors which include distorted lattice parameter and the size of nanomaterial while the developed stepwise regression offers empirical relationship with significant deviation due to insufficient strength in addressing the nonlinear relationship existing between the descriptors and specific surface area.

Support vector regression (SVR) belongs to the class of intelligent algorithms with fast computing potentials and excellent efficiency in addressing complex regression problems $[21,22]$. The algorithm is formulated using structural risk minimization principle and minimizes the empirical error through epsilon-insensitive loss function control [23-25]. Statistical learning theory upon which the algorithm forms the basis helps in error margin customization through hyperplane maximization. These features have made practical application of SVR algorithm in addressing real-life challenges and problems inevitable in various field of study [26-29]. The hyperparameters contained in SVR algorithm control the precision and accuracy of SVR-based algorithm and can be altered through various means which include grid search approach, manual search approach, or metal-heuristic approach [30, 31]. Apart from robustness, precision, and avoidance of local minimal while using metal-heuristic hyperparameter optimization, time conser- vation is also a plus to metal-heuristic approach of hyperparameter selection. The metal-heuristic algorithm implemented in this work is genetic algorithm with characteristics of fast convergence and avoidance of premature convergence [32].

Stepwise regression (STWR) is a regression algorithm through which functions and equations that directly link the descriptors with the desired target are generated through either forward selection addition process or backward deletion procedures $[33,34]$. It weighs the significance of every descriptors based on some defined criteria before inclusion in the regression function. The algorithm also allows implementation of high-order polynomials as well as interactions of descriptors into regression function. The uniqueness of this algorithm has enjoyed a wide range of real life applications in science and engineering field [35-39]. This work also develops and implements STWR algorithm for modeling the specific surface area of doped spinel ferrite nanomaterials using lattice parameter and the size of nanomaterials as descriptors.

The road map for the remaining part of the manuscript is organized as follows: Section 2 presents mathematical formulation of support vector regression algorithm, stepwise regression algorithm, and physical principles governing the operation of population-based optimization genetic algorithm. Section 3 presents the computational hybridization of support vector regression and genetic algorithm as well as the descriptions with details of data acquisition. The results are presented and discussed in Section 4 of the manuscript with inclusion of the results of algorithm comparison, while Section 5 concludes and presents the summary of the main findings of the research work.

\section{Mathematical Background of the Developed Algorithms}

The mathematical formulation of the intelligent support vector regression algorithm is presented in this section coupled with the description of genetic algorithm. The section also contains the description of the implemented stepwise regression algorithm.

2.1. Support Vector Regression Intelligent Algorithm. Consider $\kappa$ number of samples of data set defined as $\left(\iota_{j}, S_{j}^{*}\right)$, where $j=1,2, \cdots, \kappa \subset \mathbb{R}^{d}$. The estimated specific surface area of doped spinel ferrite nanomaterials using the proposed support vector regression- (SVR-) based model is represented by $S$ while the measured values of specific surface area from which patterns are to be acquired and drawn are represented by $S_{j}^{*}$. The descriptors to the proposed model which include the size of doped spinel ferrite nanomaterials and lattice distortion as measured by the lattice parameter are represented in regression equation as $\iota_{j}$. The support vector regression function is presented in Equation (1) [40, 41].

$$
S=\left\langle\delta, \iota_{j}\right\rangle+\rho
$$


where $S_{j} \in \mathbb{R}^{d}, \iota_{j} \in \mathbb{R}^{d}$, and $\rho$ is the bias of the regression function, and $\delta$ is the weight vector to be determined and optimized within support vector regression formulation.

Euclidean norm $\|\delta\|$ minimization in actualization of SVR objectives requires minimization of Equation (2) subjected to conditions and constraints itemized in Equation (3).

$$
\frac{\|\delta\|^{2}}{2}+C \sum_{j=1}^{\kappa}\left(\psi_{j}+\psi_{j}^{*}\right)
$$

where $C$ is the penalty factor that strongly influence the performance of the model.

$$
\begin{gathered}
S_{j}^{*}-\left\langle\delta, \iota_{j}\right\rangle-\rho \leq \varepsilon+\psi_{j} \\
\left\langle\delta, \iota_{j}\right\rangle+\rho-S_{j}^{*} \leq \varepsilon+\psi_{j}^{*} \\
\psi_{j}, \psi_{j}^{*} \geq 0
\end{gathered} .
$$

Modification of complex optimization problem contained in Equation (2) includes nonzero positive slack variables $\left(\psi_{j}\right.$, $\psi_{j}^{*}$ ) that have potentials of controlling further possible constraints that might hinder actualization of error minimization beyond the epsilon $\varepsilon$ threshold $[42,43]$. Introduction of Lagrange multipliers $\left(\chi, \chi^{*}\right)$ enhances dual problem transformation which aims at minimizing Equation (4) subjected to conditions presented in Equation (5).

$$
-\frac{1}{2} \sum_{j, 1=1}^{\kappa}\left(\chi_{j}-\chi_{j}^{*}\right)\left(\chi_{i}-\chi_{i}^{*}\right) \gamma\left(\iota_{j}, \iota_{i}\right)-\varepsilon \sum_{j=1}^{\kappa}\left(\chi_{j}+\chi_{j}^{*}\right)+\sum_{j=1}^{\kappa}\left(\chi_{i}-\chi_{i}^{*}\right),
$$

$$
\sum_{j=1}^{\kappa}\left(\chi_{j}-\chi_{j}^{*}\right)=0, \quad \chi_{j}, \chi_{j}^{*} \in[0, C] .
$$

$\chi$ and $\chi^{*}$ are Lagrange multipliers.

It should be noted that $\gamma\left(\iota_{j}, \iota_{i}\right)$ in Equation (4) represents kernel function which can be polynomial, sigmoid, Gaussian, or other viable functions with distinct features. The function that best mapped the lattice distortion and size of nanoparticles to high feature space is Gaussian function presented in Equation (6) [44].

$$
\gamma\left(\iota_{j}, \iota_{i}\right)=\left\langle\iota_{j}, \iota_{i}\right\rangle=\exp \left(\omega\left\|\iota_{j}-\iota_{i}\right\|^{2}\right)
$$

where $\omega$ is the kernel parameter.

The final regression function after transformation is presented in Equation (7).

$$
S(\iota)=\sum_{j=1}^{\kappa}\left(\chi_{j}-\chi_{j}^{*}\right) \gamma\left(\iota_{j}, \iota\right)+\rho .
$$

The kernel parameter $(\omega)$ of the chosen kernel function, the maximum allowable deviation $(\varepsilon)$ of the estimated specific surface area from the measured value, and the penalty factor are optimized using genetic algorithm in this research work.

2.2. Genetic Algorithm. Genetic algorithm belongs to the class of well explored evolutionary optimization algorithm which mimics and replicates natural evolution $[45,46]$. Its development and implementation might be in binary or continuous form depending on the nature of the optimization problem to be handled since binary type caters for discrete space together with continuous space. Three operators that definitely feature in genetic algorithm principles include selection, crossover, and mutation. Before selecting best candidate through fitness function evaluation, a population matrix needs to be generated randomly and houses probable solutions within the search space $[47,48]$. Crossover operation produces offsprings from the defined probable solutions called parents after the implementation of selection operator. Mutation operation aids in genetic diversity maintenance and prevents local minimal convergence. The processes are repeated over a defined number of generations until stopping conditions are satisfied. Elitism inscribes robustness and effectiveness into genetic algorithm and enhances the optimality of the algorithm [49], although canonical genetic algorithm does not include elitism as an operator and among its functions is prevention of the best solution from undergoing mutation process. This helps in transference and preserving of best solutions from one generation to the subsequent generations.

2.3. Mathematical Background of Stepwise Regression. Stepwise regression is a class of linear regression method which utilizes iterative construction of regression function in a step by step manner $[33,34]$. Stepwise regression equation is presented in matrix form as shown in Equation (8) in which $\boldsymbol{X}$ and $\boldsymbol{\beta}$, respectively, represent descriptor vector and coefficient vector of $\boldsymbol{X}$.

$$
y=X \beta+\varepsilon,
$$

where $\boldsymbol{y}=\left[y_{1}, y_{2}, y_{3} \cdots y_{k}\right]^{T}, \boldsymbol{X}=\left[x^{T}{ }_{1}, x^{T}{ }_{2}, x^{T}{ }_{3}, x^{T}{ }_{k}\right]^{T}, \boldsymbol{\beta}=$ $\left[\beta_{0}, \beta_{1}, \beta_{2}, \cdots . . \beta_{n-1}\right]$, and $\varepsilon=\left[\varepsilon_{1}, \varepsilon_{2}, \varepsilon_{3} \cdots \varepsilon_{k}\right]^{T}$.

It should be noted that the length of $\boldsymbol{\beta}$ is the length of descriptors which is two (the lattice parameter of doped spinel ferrite nanomaterials and the size of the nanomaterials) in this problem. Stepwise regression algorithm aims at iteratively and recursively determining the link between the descriptors and the target by weighing the contribution of each of the descriptors [50]. However, metrics adopted in adding and deleting descriptors include adjusted $R^{2}$, Bayesian Information Criteria, Akaike Information Criteria, and Sum of Squared Error. The estimation strength of each of these criteria is judged using root mean square error (RMSE) of the tested samples. The procedures for adding and deleting descriptors are termed forward selection and backward elimination, respectively [33]. The choice of either of these methods does not in any way often affect the accuracy of the resulting regression function. However, one may save computational time than the other. In forward selection process, one descriptor is selected and added to regression 
TABLE 1: Statistical analysis of the spinel ferrite nanomaterial dataset.

\begin{tabular}{lccccc}
\hline & Maximum & Average & Minimum & Standard deviation & Correlation coefficient \\
\hline Lattice parameter a $(\AA \dot{A})$ & 8.7260 & 8.4360 & 8.2610 & 0.1134 & -0.2658 \\
Size of nanomaterial $(\mathrm{nm})$ & 51.7030 & 27.0510 & 6.7000 & 11.6199 & -0.2667 \\
Specific surface area $\left(\mathrm{M}^{2} / \mathrm{g}\right)$ & 92.7000 & 37.1288 & 0.4000 & 21.5535 & 1 \\
\hline
\end{tabular}

function in the first step before adding the second descriptor after ensuring that the best fit is provided by its addition. The process continues until all the descriptors are added after satisfying some requirement such as adjusted $R^{2}$, Bayesian Information Criteria, Akaike Information Criteria, and Sum of Squared Error. In backward elimination process, the development of regression function begins with inclusion of all the descriptors while one by one elimination follows after inability to meet up with the defined requirement and criteria. The choice of stepwise regression becomes significant in the addressed problem to ascertain the potential of nonlinear model in addressing and establishing the relationship between lattice parameter, nanomaterial size, and specific surface area of doped spinel ferrite nanomaterials.

\section{Computational Methodology of the Developed Model}

The details of the computational hybridization of support vector regression and genetic algorithm are presented here. This section also contains the details of the employed dataset and the results of the initial statistical analysis performed on the dataset.

\subsection{Acquisition and Description of Spinel Ferrite} Nanomaterial Dataset. The lattice parameter of doped spinel ferrite nanomaterials obtained from X-ray diffraction analysis serves as the descriptor to the developed hybrid GBSVR model. The model also factors in the size of nanomaterial as its descriptor for estimating the specific surface area of spinel ferrite nanomaterial. The lattice distortion and the size of the nanomaterials with their corresponding specific surface area for forty spinel ferrite nanomaterials doped with different nanoparticles are extracted from the literature for the model development $[5,7,8,18,51-55]$. The lattice strain as well as structural distortion in lattice structure of the parent spinel ferrite nanomaterial resulted from the introduced dopants definitely alters the material specific surface area. Table 1 presents the outcomes of statistical parameters evaluated on the dataset.

Statistical parameters are evaluated on the lattice parameter, size of nanomaterial, and the measured specific surface area of doped spinel ferrite nanomaterials. The presented average (mean) values in the table as well as maximum and minimum values collectively reflect the overall content of the employed dataset. The significance of the standard deviation analysis presented in the table is to give a useful insight about the dispersion of the values of specific surface area and descriptors for different spinel ferrite nanomaterials doped with external materials. The correlation coefficients between the measured specific surface area and each of the descriptors reveal the potentialities of nonlinear model in establishing the relationship between specific surface area and descriptors. This gives an insight that the proposed hybrid genetic algorithm-based support vector regression (GBSVR) has potentials to outperform the proposed stepwise regression (STWR) that fails to incorporate nonlinearity in its operational modalities.

\subsection{Computational Development of Proposed Hybrid Genetic} Algorithm-Based Support Vector Regression. Strong dependence of the performance of support vector regressionbased model on the combinatory choice of hyperparameters necessitates proper tuning and optimization of hyperparameters which include the maximum allowable deviation (epsilon), penalty factor, and kernel parameter of the best kernel function. The computational development of the proposed hybrid GBSVR model was conducted within computing environment of MATLAB. Dataset partitioning was carried out into training and testing set using $8: 2$ ratio while dataset randomization precedes data separation. Randomization promotes even, uniform, and just distribution of data points so that the possibility of validating and testing model on tasks that the model fails to learn due to insufficient data dispersion is prevented. With forty total number of doped spinel ferrite nanomaterials investigated, thirty-two doped compounds were employed in pattern acquisition during training stage, while the remaining eight doped compounds were utilized in evaluating the future performance of the model. The following procedures summarize the description of support vector regression hybridization with the genetic algorithm for performance enhancement.

Step 1. Population matrix: every chromosome in the population matrix carries information regarding the epsilon, penalty factor, and kernel parameter in a known and defined order. The kernel parameter is contained in the chosen kernel function and helps in smooth transformation of data to feature space where the construction of regression function takes place. The search space for the epsilon was set as 0.4 and 0.1 for upper and lower bound while setting outside this range resulted into perpetual running of the code. The kernel parameter ranges from 0.4 to 0.1 while the penalty factor was maintained between 1000 and 1 . The right choice of hyperparameter range strengthens the exploitation and exploration capacities of the model and leads to appreciable time conservation.

Step 2. Chromosome fitness calculation: determination of chromosome fitness involves the implementation of SVR algorithm with training and testing set of data. Nonlinear 


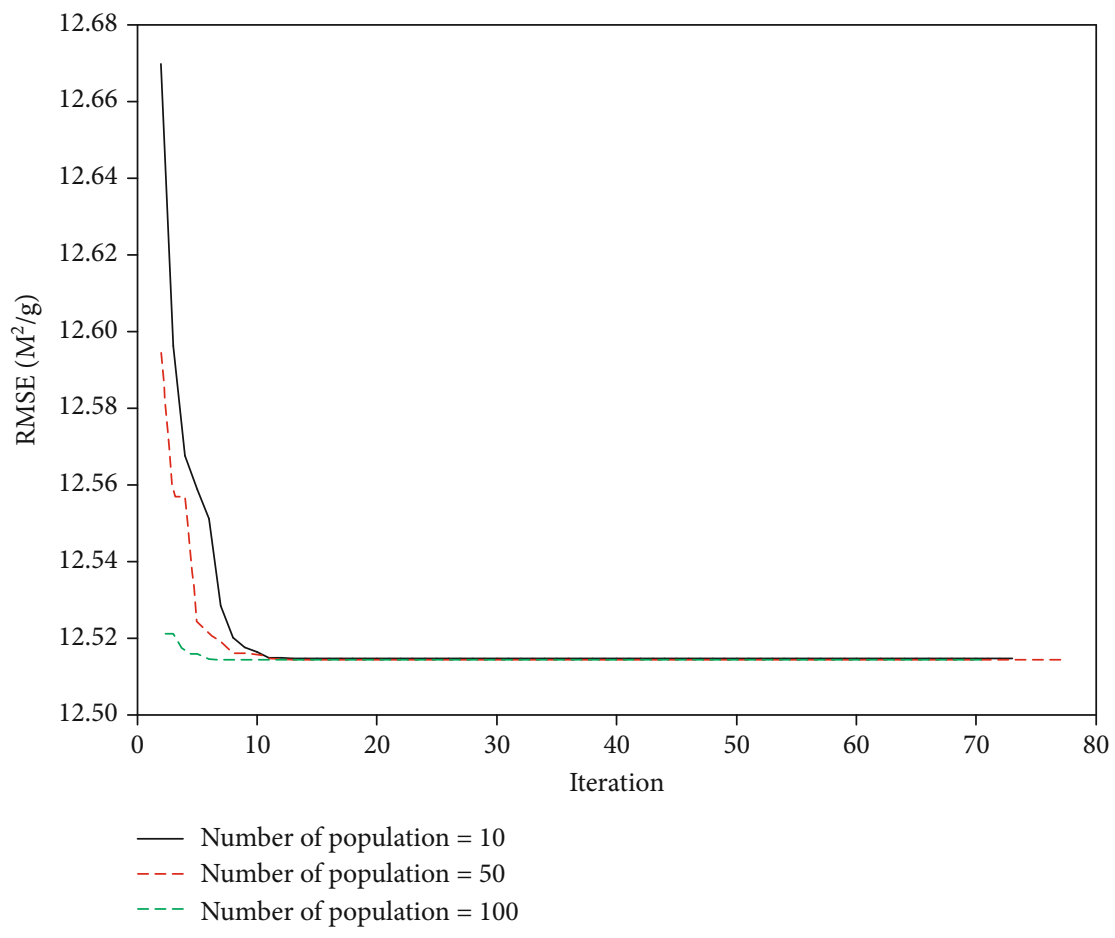

FIgURE 1: Convergence of GBSVR model to the number chromosomes in population matrix.

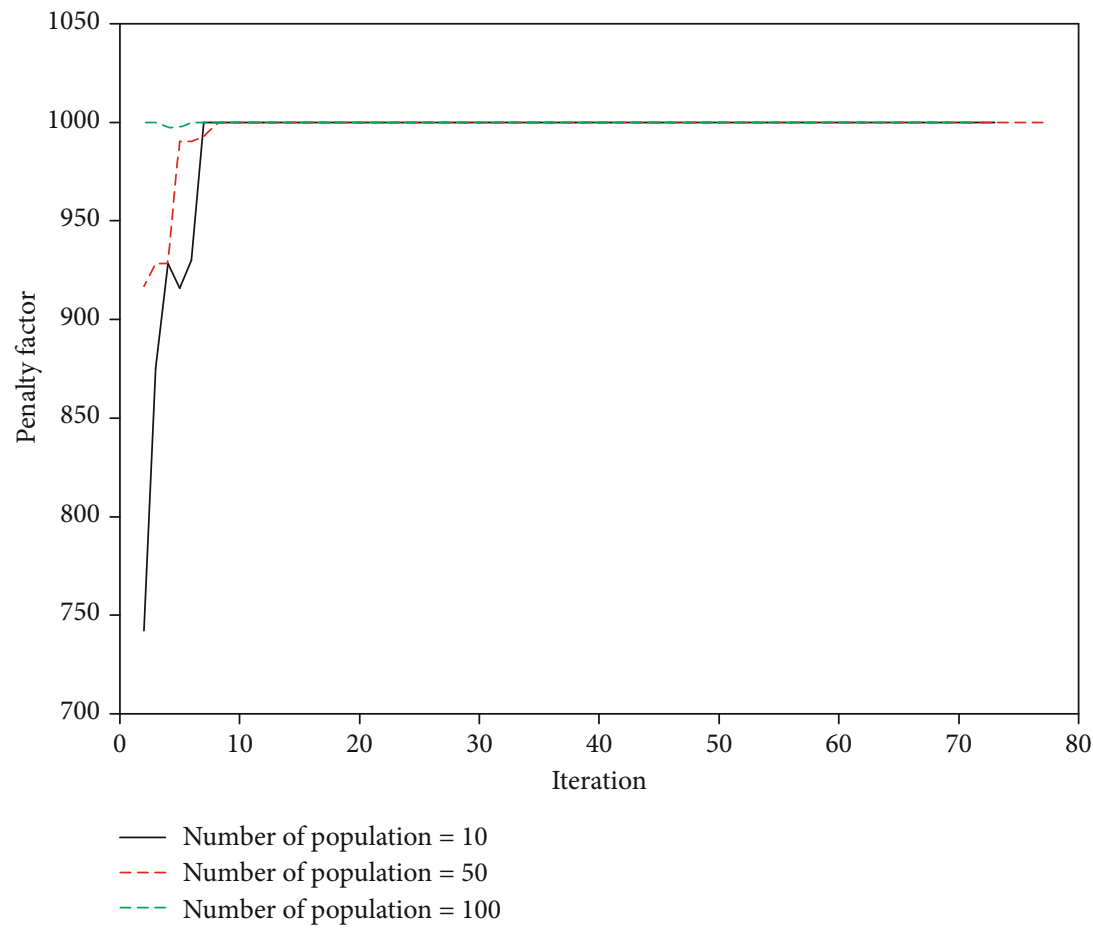

FIgURE 2: Sensitivity of GBSVR to penalty factor for different population matrix.

mapping function was chosen among the pools of function, while the chosen function combines with each of the chromosome to access the encoded values of the kernel parameter, penalty factor, and epsilon. The chromosome integrated mapping function takes in the training set of data for sup- port vector generation. The hyperplane parameter is set at E-7, while each SVR algorithm trained with chromosome integrated mapping function was evaluated and accessed using root mean square error (RMSE) on the testing set of data coupled with the saved support vectors during training. 


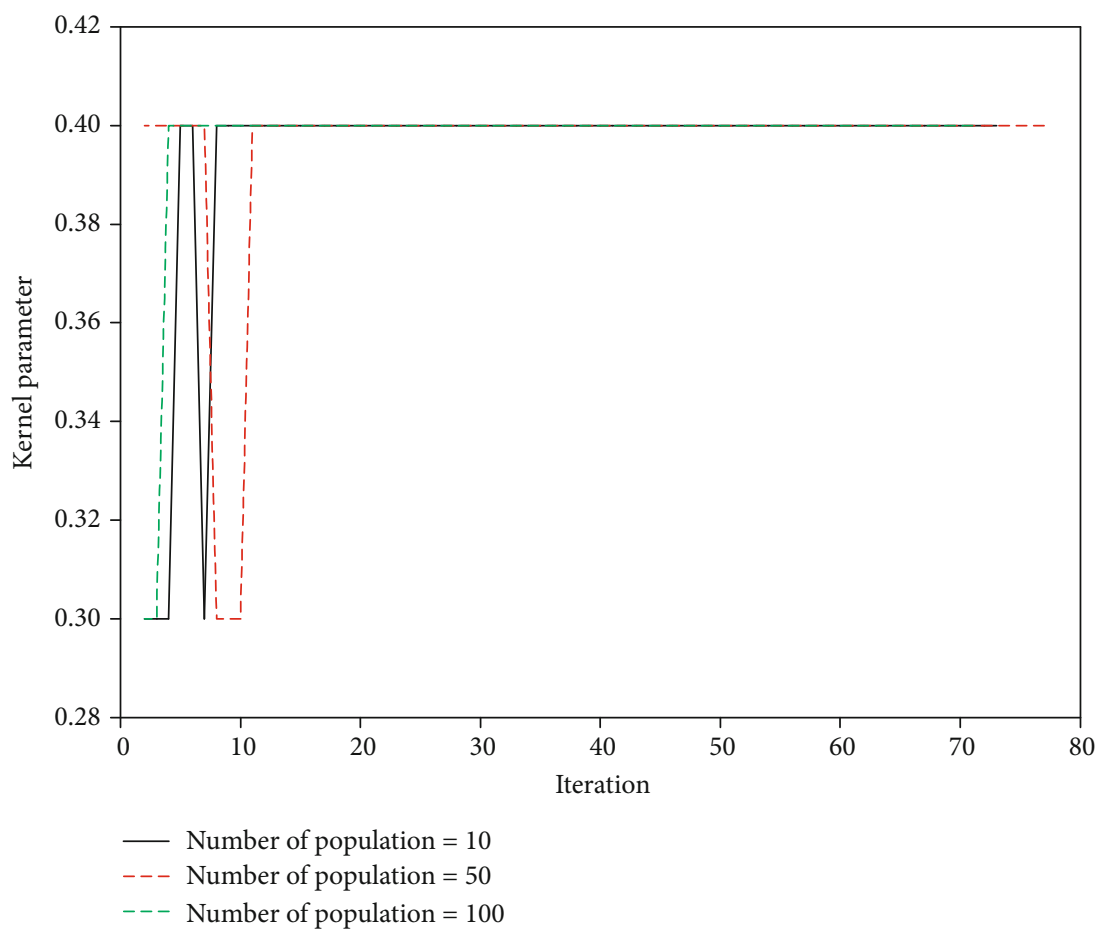

FIgURE 3: Sensitivity of GBSVR to Gaussian kernel parameter for different population matrix.

The chromosome with the lowest value of RMSE during testing stage is most fit, while other chromosomes were ranked in accordance to their fitness.

Step 3. Reproduction operation: reproduction involves selection of best chromosome with distinct qualities (usually characterized with lowest RMSE value) for transition to the next generation. In order to enhance fruitful and desired transition, a tournament selection operation with probability of 0.8 was implemented. Tournament selection involves random selection of few chromosomes from the whole population for tournament while the winners of the tournament transit to crossover stage of operation.

Step 4. Crossover stage: in crossover stage, new offsprings with desired qualities and potentials are formed from portions and subsequences of two parents. The probability value was set at 0.65 in order to achieve effective subsequences transfer from parent to the offsprings.

Step 5. Mutation stage: Altering positions that are randomly chosen within the chromosome is controlled through mutation operation, and the probability value was set at 0.009 in this present work.

Step 6. Stopping condition: the algorithm repeats the cycle between Step 1 and Step 5 until the same value of fitness is attained for consecutive sixty iterations.
TABLE 2: Results of genetic optimization algorithm.

\begin{tabular}{lc}
\hline Model parameter & Optimum value \\
\hline Penalty factor & 1000 \\
Number of chromosomes & 50 \\
Epsilon & 0.1 \\
Hyperparameter lambda & E-7 \\
Kernel parameter & 0.4 \\
Kernel function & Gaussian \\
\hline
\end{tabular}

\section{Results and Discussion}

The outcomes of the developed hybrid GBSVR model are discussed in this section. The performance of the developed GBSVR model is compared with that of STWR using different evaluation parameters. The sensitivity and convergence of the developed hybrid GBSVR model to the hyperparameter and the content of the population matrix is presented in this section. Comparison of the estimated specific surface area of spinel ferrite nanomaterials with the measured values for various dopants incorporation is also presented in this section.

4.1. Sensitivity and Convergence of the Developed Hybrid GBSVR Model. The convergence of the developed hybrid GBSVR model at various iteration with different number of chromosomes in population matrix is presented in Figure 1.

The exploitation as well as exploration strength of the developed hybrid model is optimized as convergence is attained. It was observed from the convergence graph 


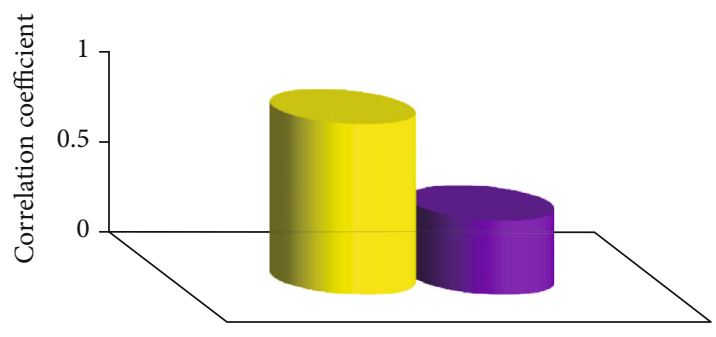

Training dataset

GBSVR

- STWR

(a)

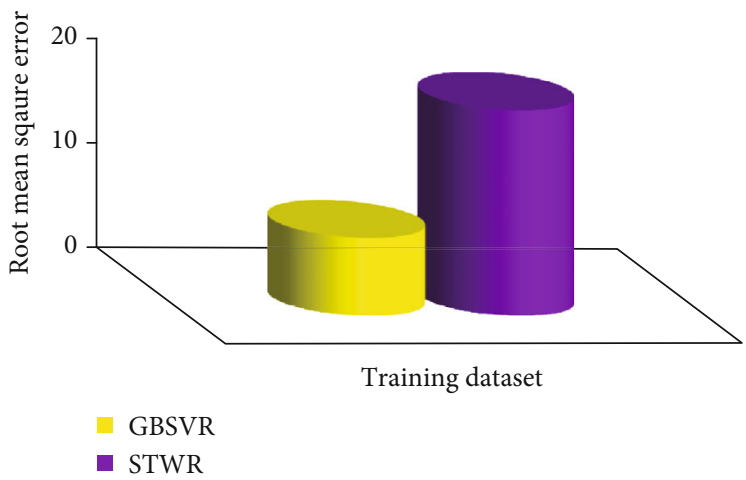

(c)

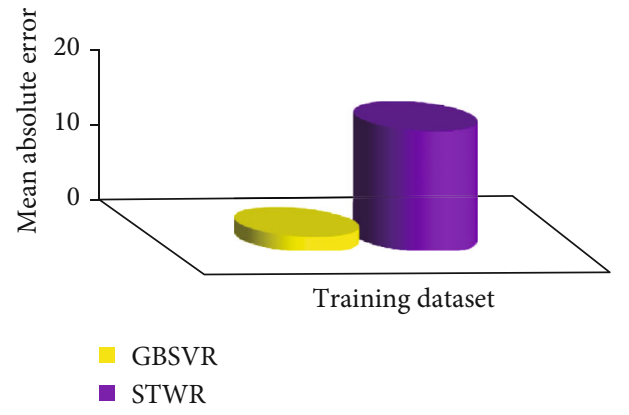

(e)

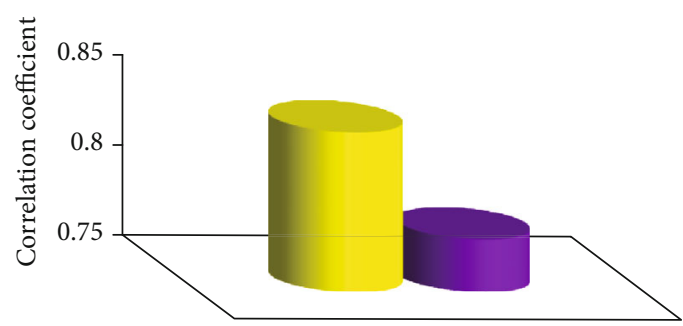

Testing dataset

GBSVR

- STWR

(b)

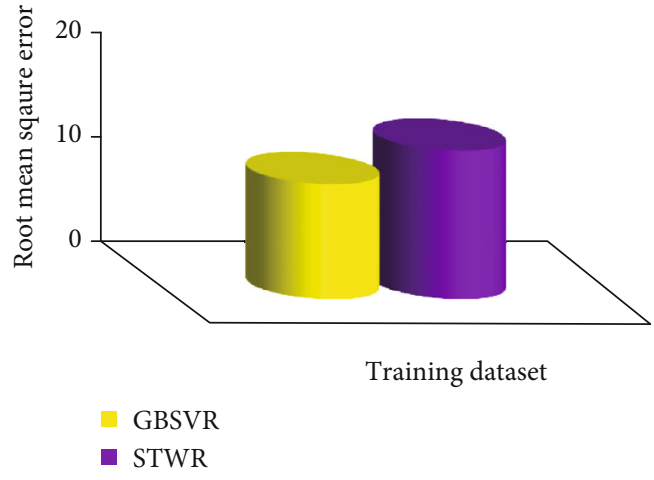

(d)

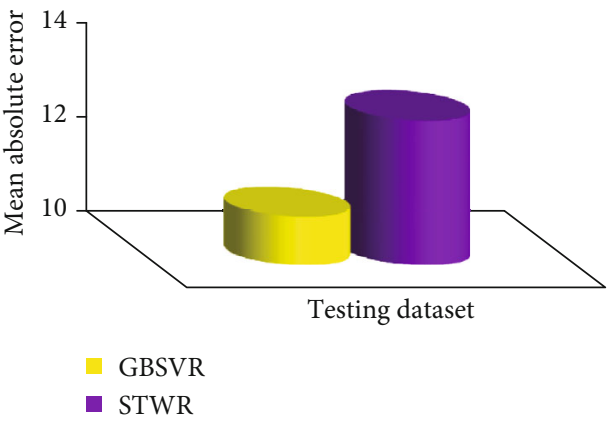

(f)

FIGURE 4: Comparing the performance of the developed models during training and testing phases using different parameters for performance evaluation.

presented in Figure 1 that the developed model is robust and shows the same point of convergence for different number of chromosomes in the population matrix. The optimum population matrix was set at fifty from the obtained global solution convergence. The sensitivity of the developed GBSVR model to the penalty hyperparameter factor is presented in Figure 2 at various values of chromosomes in the population matrix.

When ten numbers of chromosomes are navigating the search space, the penalty factor initializes at a value of 750 and increases steadily over ten iterations before begins the convergence. For fifty numbers of chromosomes, the chromosomes initialize at a value higher than the initial value of ten chromosomes, and similar convergence is attained. The convergence presented in Figure 2 shows that the global solution is well explored when there are many chromosomes
TABLE 3: Performance of the developed model at stages of model development.

\begin{tabular}{lcccc}
\hline & \multicolumn{2}{c}{ GBSVR } & \multicolumn{2}{c}{ STWR } \\
& Training & Testing & Training & Testing \\
\hline CC & 0.944 & 0.8452 & 0.41298 & 0.78174 \\
RMSE & 7.4631 & 12.5351 & 19.61918 & 16.21857 \\
MAE & 2.1146 & 11.0318 & 16.23133 & 13.05923 \\
\hline
\end{tabular}

within the search space. The sensitivity of the developed model with respect to the kernel parameter is presented in Figure 3. Similar trend is attained, and the model converged to global solution with 0.4 value of kernel parameter for Gaussian mapping function. The results of genetic optimization algorithm for each of the optimized hyperparameter are presented in Table 2. 


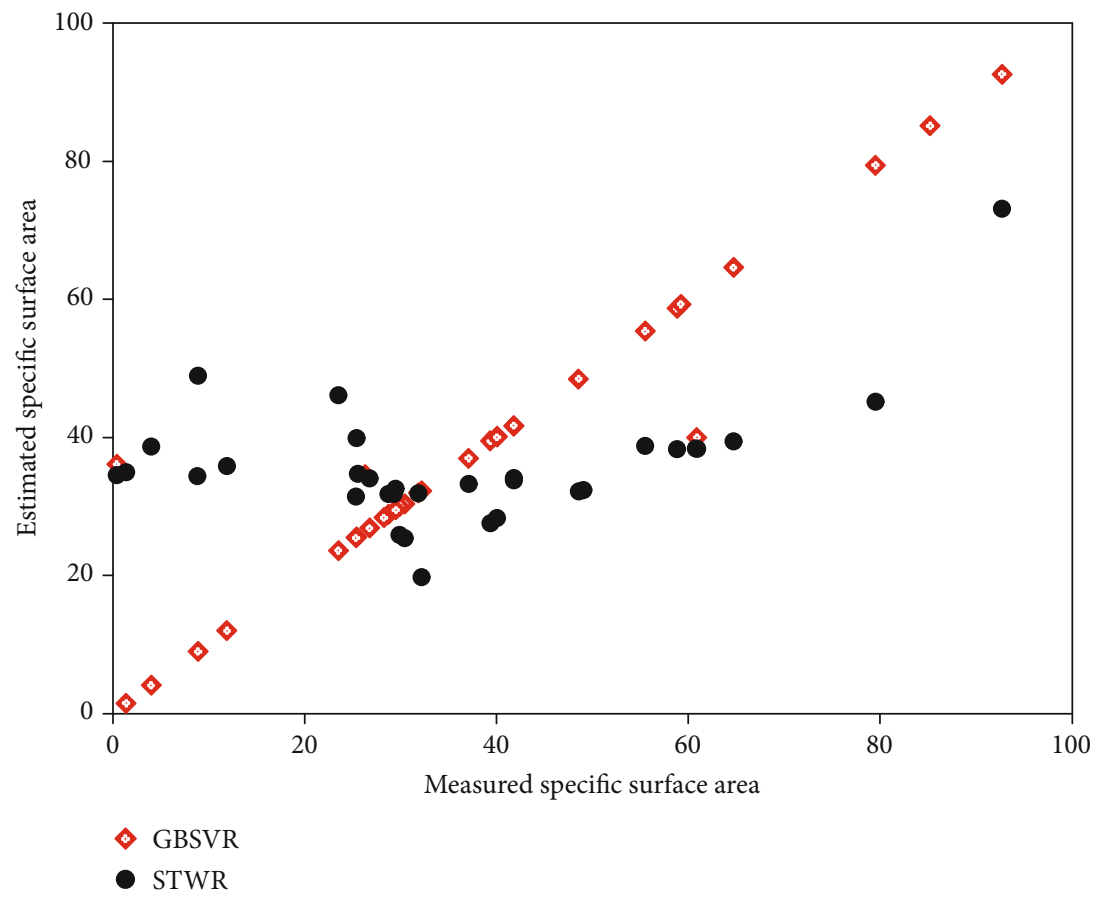

(a)

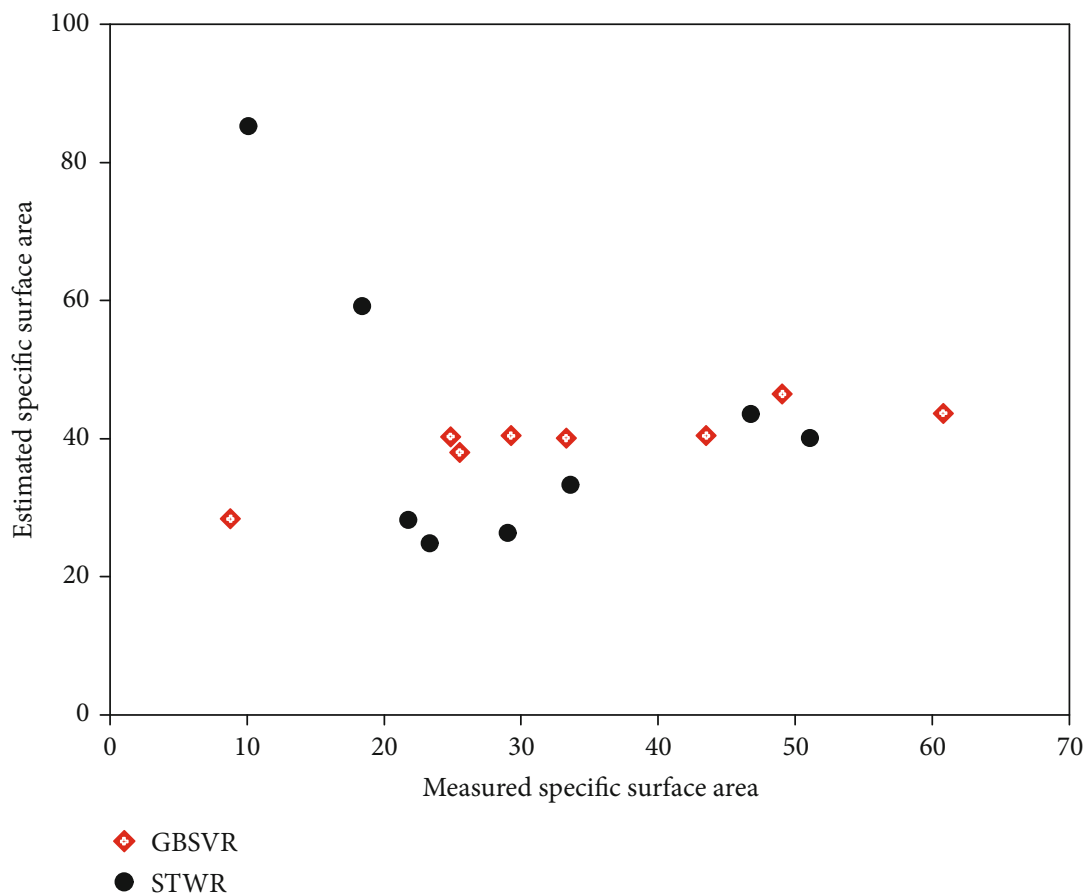

(b)

FIgURE 5: Correlation cross-plot between the measured specific surface area and estimated values for different phase of the developed models.

4.2. Evaluation of the Performance of the Developed Models. The performance of the developed GBSVR and STWR models are compared during the training and testing phases of model development using correlation coefficient (CC) between measured and estimated specific surface area, mean absolute error (MAE), and root mean square error (RMSE). The outcomes of the comparison are presented in Figure 4.
The comparison on the basis of correlation coefficient on training dataset presented in Figure 4(a) shows that the developed hybrid GBSVR model displays enhanced performance over the developed STWR-based model with performance improvement of 56.25\%, while the testing stage presented in Figure 4(b) shows performance superiority of 7.508\%. Using RMSE for comparing the performance as 


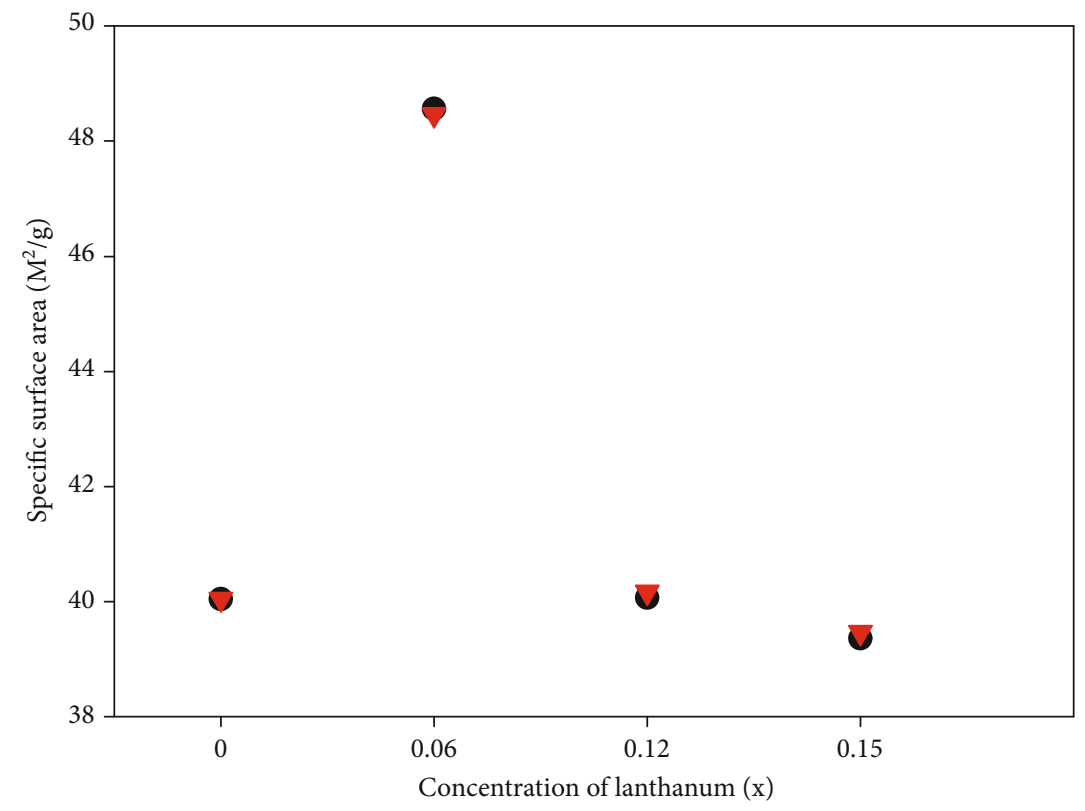

Measured specific surface area

$\nabla$ Estimated specific surface area using the developed GBSVR model

FIGURE 6: Effect of lanthanum dopants on specific surface area of $\mathrm{Ni}_{0.15} \mathrm{Cu}_{0.25} \mathrm{Co}_{0.35} \mathrm{Fe}_{2-x} \mathrm{La}_{x} \mathrm{O}_{4}$ spinel ferrite nanomaterial.

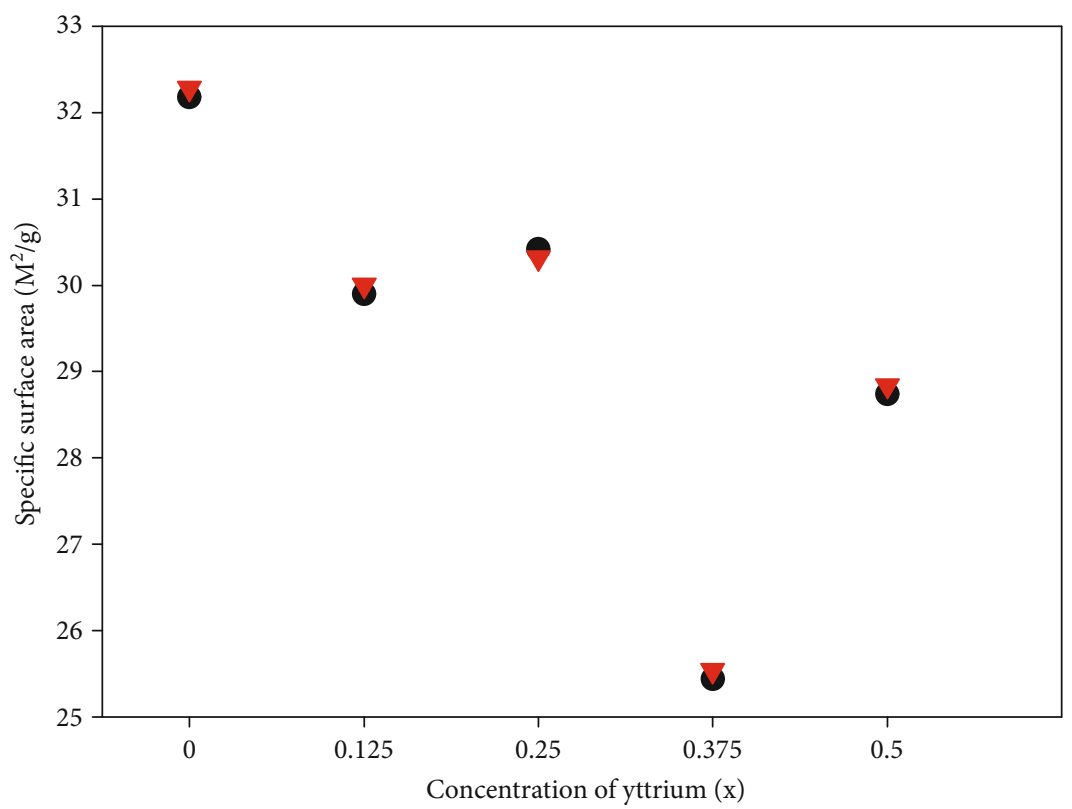

- Measured specific surface area

$\nabla$ Estimated specific surface area using the developed GBSVR model

FIgURE 7: Significance of yttrium nanoparticles on specific surface area of $\mathrm{Cd}_{1-x} \mathrm{Y}_{x} \mathrm{Fe}_{2} \mathrm{O}_{4}$ spinel ferrite nanomaterial.

presented in Figures 4(c) and 4(d), the developed GBSVR model outperforms STWR model with performance improvement of $61.96 \%$ and $22.68 \%$ for training and testing set of data, respectively. Similar trend of performance improvement of $86.97 \%$ and $15.52 \%$ was, respectively, obtained while comparing the developed GBSVR and STWR models on the basis of mean absolute error (MAE) as presented in Figures 4(e) and 4(f). Table 3 contains the results of the performance measuring metrics for training and testing dataset.

Correlation cross-plots between the measured specific surface area of doped spinel ferrite nanomaterials and the predicted values are presented in Figure 5 for training (Figure 5(a)) and testing (Figure 5(b)) set of data. The precision of the model can be judged from correlation cross-plots through alignment of data points. The results of the 


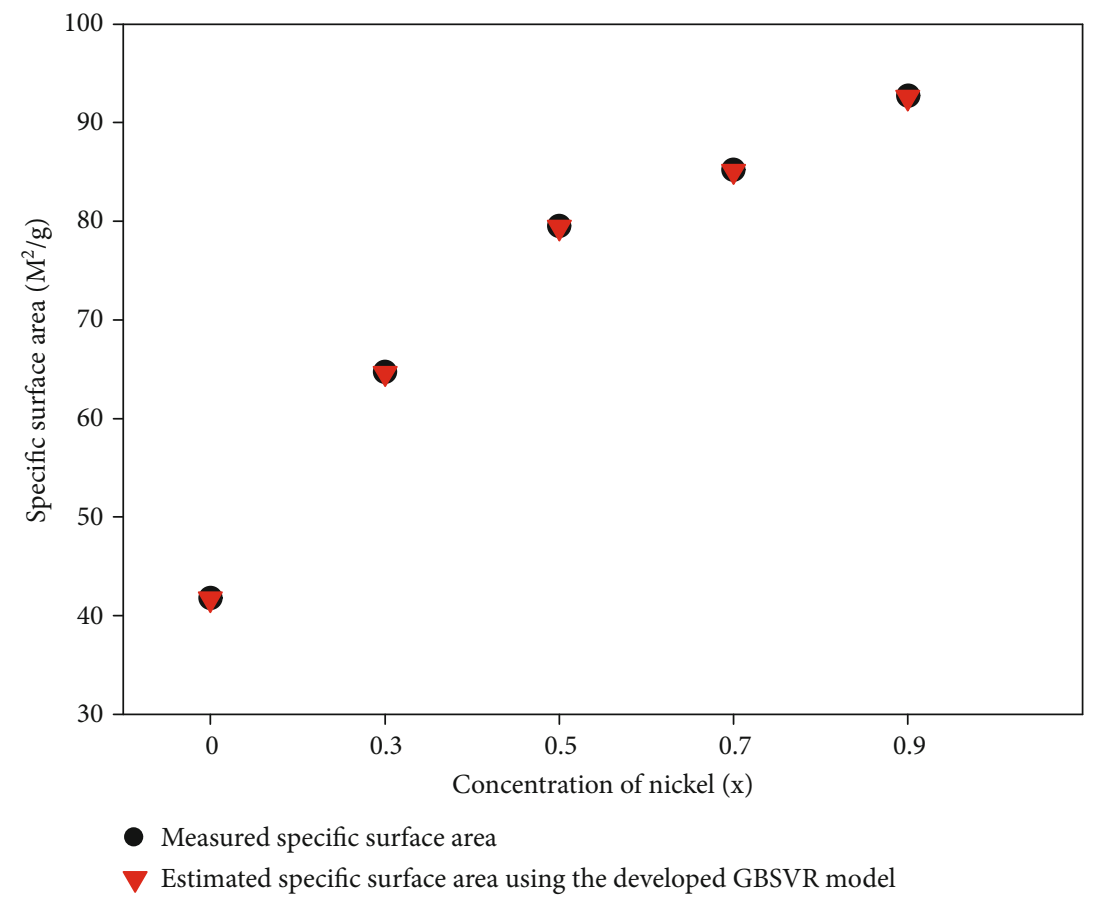

FIGURE 8: Doping effect of nickel on specific surface area of $\mathrm{Co}_{1-x} \mathrm{Ni}_{x} \mathrm{Fe}_{2} \mathrm{O}_{4}$ spinel ferrite nanomaterial.

developed GBSVR model are well aligned during training and testing stages, while dispersion and deviations characterize the outcomes of the developed STWR model. The observed poor performance of the developed STWR model can be attributed to the inability of STWR model to adequately describe the nonlinear connections between the lattice parameter of doped spinel ferrite nanomaterials, the particle size, and specific surface area.

4.3. Influence of Lanthanum Incorporation on Specific Surface Area of $\mathrm{Ni}_{0.15} \mathrm{Cu}_{0.25} \mathrm{Co}_{0.35} \mathrm{Fe}_{2 x} \mathrm{La}_{x} \mathrm{O}_{4}$ Spinel Ferrite Nanomaterial Using the Developed Hybrid GBSVR Model. Incorporation of lanthanum nanoparticles into the parent crystal lattice of $\mathrm{Ni}_{0.15} \mathrm{Cu}_{0.25} \mathrm{Co}_{0.35} \mathrm{Fe}_{2 x} \mathrm{La}_{x} \mathrm{O}_{4}$ spinel ferrite nanomaterial has been modeled using developed GBSVR, and the comparison between the measured and estimated specific surface area is presented in Figure 6. The effect of grain boundary deposition of lanthanum is initially observed from increase in specific surface area when the concentration of lanthanum nanoparticles is 0.06 . Subsequently, after this concentration, the lanthanum nanoparticles get diffused into the parent crystal structure which leads to decrease in the value of specific surface area [5]. Few particles of iron $\left(\mathrm{Fe}^{3+}\right)$ in tetrahedral sites were replaced with lanthanum $\left(\mathrm{La}^{3+}\right)$ particles in octahedral sites, while this charge diffusion is responsible for the observed variation in specific surface area. The predicted specific surface area using the developed GBSVR model agree excellently well with the measured values [5].

4.4. Tailoring Specific Surface Area of $\mathrm{Cd}_{1-x} \mathrm{Y}_{x} \mathrm{Fe}_{2} \mathrm{O}_{4}$ Spinel Ferrite Nanomaterial through Yttrium Dopants Using the Developed GBSVR Model. Figure 7 presents the effect of yttrium nanoparticles incorporation on specific surface area of doped $\mathrm{Cd}_{1-x} \mathrm{Y}_{x} \mathrm{Fe}_{2} \mathrm{O}_{4}$ spinel ferrite nanomaterial using the developed GBSVR model. Comparison of the measured specific surface area with the estimated values using the developed GBSVR model as presented in Figure 7 shows excellent agreement [52]. The observed variation of specific surface area due to yttrium incorporation can be inferred from the fact that substitution with higher ionic radius creates spaces in the configurations of spinel ferrite material. Cationic radius characterizing the nanoparticles substituted for yttrium surfers no alteration since the octahedral site of iron remains unchanged.

4.5. Importance of Nickel Nanoparticles in Enhancing Specific Surface Area of Doped $\mathrm{Co}_{1-x} \mathrm{Ni}_{x} \mathrm{Fe}_{2} \mathrm{O}_{4}$ Spinel Ferrite Nanomaterial Using the Developed Hybrid GBSVR Model. Inclusion of nickel nanoparticles in the crystal structure of $\mathrm{Co}_{1-x} \mathrm{Ni}_{x} \mathrm{Fe}_{2} \mathrm{O}_{4}$ spinel ferrite nanomaterial further reveals the significance of cation distribution on the physical properties of spinel ferrite nanomaterials especially the specific surface area. $\mathrm{As} \mathrm{Fe}^{3+}$ ions is distributed among the available tetrahedral and octahedral sites, the incorporated nickel $\left(\mathrm{Ni}^{2+}\right)$ nanoparticle ions exist within tetrahedral sites while cobalt $\left(\mathrm{Co}^{2+}\right)$ ions reside in octahedral sites [8]. The comparison presented in Figure 8 shows that the experimental trend of nickel incorporation in lattice structure of $\mathrm{Co}_{1-x} \mathrm{Ni}_{x} \mathrm{Fe}_{2} \mathrm{O}_{4}$ spinel ferrite nanomaterial is well captured by the developed hybrid GBSVR model [8].

\section{Conclusion}

Specific surface area of doped spinel ferrite nanomaterials is modeled in this work using stepwise regression (STWR) algorithm and hybrid genetically based support vector regression (GBSVR) algorithm. The descriptors to the 
developed models include the lattice distortion due to dopant inclusion in lattice structure of spinel ferrite nanomaterials and the size of nanomaterials. The models were developed and validated using forty different spine ferrite nanomaterials subjected to incorporation of different dopants at various experimental conditions. The developed GBSVR model outperforms STWR model when evaluated using mean absolute error, correlation coefficient, and root mean square error metrics. The developed GBSVR model investigates the significance of lanthanum, yttrium, and nickel nanoparticle inclusions in different matrixes of spinel ferrite nanomaterials and the outcomes of the developed model agree excellently well with the measured specific surface areas. The outstanding performance demonstrated by the developed model is of enormous significance in tailoring the specific surface area of spinel ferrite nanomaterials to desired values needed for specific application with resources conservation and circumvention of experimental stress.

\section{Data Availability}

The raw data required to reproduce these findings are available in the cited references in Section 3.1 of the manuscript.

\section{Conflicts of Interest}

The authors declare that they have no conflicts of interest.

\section{References}

[1] M. N. Akhtar, M. Yousaf, Y. Lu et al., "Physical, structural, conductive and magneto-optical properties of rare earths ( $\mathrm{Yb}, \mathrm{Gd}$ ) doped Ni-Zn spinel nanoferrites for data and energy storage devices," Ceramics International, vol. 47, no. 9, pp. 11878-11886, 2021.

[2] Y. Slimani, B. Unal, M. A. Almessiere et al., "Investigation of structural and physical properties of $\mathrm{Eu}^{3+}$ ions substituted $\mathrm{Ni}_{0.4} \mathrm{Cu}_{0.2} \mathrm{Zn}_{0.4} \mathrm{Fe}_{2} \mathrm{O}_{4}$ spinel ferrite nanoparticles prepared via sonochemical approach," Results in Physics, vol. 17, article 103061, 2020.

[3] Y. Slimani, M. A. Almessiere, M. Sertkol et al., "Structural, magnetic, optical properties and cation distribution of nanosized $\mathrm{Ni}_{0.3} \mathrm{Cu}_{0.3} \mathrm{Zn}_{0.4} \mathrm{Tm}_{\mathrm{x}} \mathrm{Fe}_{2-\mathrm{x}} \mathrm{O}_{4}(0.0 \leq \mathrm{x} \leq 0.10)$ spinel ferrites synthesized by ultrasound irradiation," Ultrasonics, vol. 57, pp. 203-211, 2019.

[4] H. V. S. Pessoni, T. E. P. Alves, P. Banerjee, and A. F. Júnior, "Functional properties of $\mathrm{Ho}^{3+}$ substituted cobalt ferrite in the context of the reduced mass model," Physica B: Condensed Matter, vol. 575, article 411676, 2019.

[5] A. Aslam, N. A. Morley, N. Amin et al., "Study of structural, optical and electrical properties of La 3 + doped Mg 0.25," Physica B: Condensed Matter, vol. 602, article 412565, 2021.

[6] G. L. Jadhav, S. D. More, C. M. Kale, and K. M. Jadhav, "Effect of magnesium substitution on the structural, morphological, optical and wettability properties of cobalt ferrite thin films," Physica B: Condensed Matter, vol. 555, pp. 61-68, 2019.

[7] A. Rajeshwari, I. Kartharinal Punithavthy, S. Johnson Jeyakumar, N. Lenin, and B. Vigneshwaran, "Dependance of lanthanum ions on structural, magnetic and electrical of manganese based spinel nanoferrites," Ceramics International, vol. 46, no. 5, pp. 6860-6870, 2020.
[8] P. A. Vinosha, A. Manikandan, R. Ragu et al., "Impact of nickel substitution on structure, magneto-optical, electrical and acoustical properties of cobalt ferrite nanoparticles," Journal of Alloys and Compounds, vol. 857, p. 157517, 2021.

[9] M. Hao, M. Qiu, H. Yang, B. Hu, and X. Wang, "Recent advances on preparation and environmental applications of MOF-derived carbons in catalysis," Science of the Total Environment, vol. 760, p. 143333, 2021.

[10] L. Yao, H. Yang, Z. Chen, M. Qiu, B. Hu, and X. Wang, "Bismuth oxychloride-based materials for the removal of organic pollutants in wastewater," Chemosphere, vol. 273, p. 128576 , 2021.

[11] X. Liu, R. Ma, L. Zhuang et al., "Recent developments of doped g-C3N4 photocatalysts for the degradation of organic pollutants," Critical Reviews in Environmental Science and Technology, vol. 51, no. 8, pp. 751-790, 2021.

[12] R. Sharma, P. Thakur, P. Sharma, and V. Sharma, "Ferrimagnetic $\mathrm{Ni}^{2+}$ doped $\mathrm{Mg}-\mathrm{Zn}$ spinel ferrite nanoparticles for high density information storage," Journal of Alloys and Compounds, vol. 704, pp. 7-17, 2017.

[13] J. Zhang, H. Bai, S. He et al., "Effects of structure and electronic properties of spinel ferrites on their emissivity in middle and short wavebands," Journal of Solid State Chemistry, vol. 282, article 121089, 2020.

[14] A. G. Abraham, A. Manikandan, E. Manikandan et al., "Enhanced magneto-optical and photo-catalytic properties of transition metal cobalt $\left(\mathrm{Co}^{2+}\right.$ ions $)$ doped spinel $\mathrm{MgFe}_{2} \mathrm{O}_{4}$ ferrite nanocomposites," Journal of Magnetism and Magnetic Materials, vol. 452, pp. 380-388, 2018.

[15] M. Sundararajan, L. John Kennedy, P. Nithya, J. Judith Vijaya, and M. Bououdina, "Visible light driven photocatalytic degradation of rhodamine B using $\mathrm{Mg}$ doped cobalt ferrite spinel nanoparticles synthesized by microwave combustion method," Journal of Physics and Chemistry of Solids, vol. 108, pp. 61-75, 2017.

[16] M. A. Almessiere, A. Demir Korkmaz, Y. Slimani, M. Nawaz, S. Ali, and A. Baykal, "Magneto-optical properties of rare earth metals substituted Co-Zn spinel nanoferrites," Ceramics International, vol. 45, no. 3, pp. 3449-3458, 2019.

[17] Y. Slimani, H. Güngüneş, M. Nawaz et al., "Magneto-optical and microstructural properties of spinel cubic copper ferrites with $\mathrm{Li}$-Al co-substitution," Ceramics International, vol. 44, no. 12, pp. 14242-14250, 2018.

[18] S. B. Somvanshi, S. A. Jadhav, M. V. Khedkar, P. B. Kharat, S. D. More, and K. M. Jadhav, "Structural, thermal, spectral, optical and surface analysis of rare earth metal ion $\left(\mathrm{Gd}^{3+}\right)$ doped mixed $\mathrm{Zn}-\mathrm{Mg}$ nano-spinel ferrites," Ceramics International, vol. 46, no. 9, pp. 13170-13179, 2020.

[19] H. M. Gobara, I. M. Nassar, A. M. A. El Naggar, and G. Eshaq, "Nanocrystalline spinel ferrite for an enriched production of hydrogen through a solar energy stimulated water splitting process," Energy, vol. 118, pp. 1234-1242, 2017.

[20] H. Javed, A. Rehman, S. Mussadiq et al., "Reduced graphene oxide-spinel ferrite nano-hybrids as magnetically separable and recyclable visible light driven photocatalyst," Synthetic Metals, vol. 254, pp. 1-9, 2019.

[21] S. O. Olatunji and T. O. Owolabi, "Barium titanate semiconductor band gap characterization through gravitationally optimized support vector regression and extreme learning machine computational methods," Mathematical Problems in Engineering, vol. 2021, Article ID 9978384, 12 pages, 2021. 
[22] V. N. Vapnik, Statistical Learning Theory, Wiley-Interscience, New York, New York, USA, 1998.

[23] H. Tokuyama, H. Mori, R. Hamaguchi, and G. Kato, "Prediction of the lower critical solution temperature of poly $(\mathrm{N}$-isopropylacrylamide-co-methoxy triethyleneglycol acrylate) in aqueous salt solutions using support vector regression," Chemical Engineering Science, vol. 231, p. 116325, 2021.

[24] M. Liu, K. Luo, J. Zhang, and S. Chen, "A stock selection algorithm hybridizing grey wolf optimizer and support vector regression," Expert Systems with Applications, vol. 179, article 115078, 2021.

[25] S. M. I. Shamsah and T. O. Owolabi, "Newtonian mechanics based hybrid machine learning method of characterizing energy band gap of doped zno semiconductor," Chinese Journal of Physics, vol. 68, pp. 493-506, 2020.

[26] A. A. Adewunmi, S. Ismail, T. O. Owolabi, A. S. Sultan, S. O. Olatunji, and Z. Ahmad, "Hybrid intelligent modelling of the viscoelastic moduli of coal fly ash based polymer gel system for water shutoff treatment in oil and gas wells," The Canadian Journal of Chemical Engineering, vol. 97, no. 11, pp. 29692978, 2019.

[27] H. B. Adeyemo, T. O. Owolabi, M. A. Suleiman et al., "Hybrid chemometric approach for estimating the heat of detonation of aromatic energetic compounds," Heliyon, vol. 5, no. 7, p. e02035, 2019.

[28] T. O. Owolabi, K. O. Akande, S. O. Olatunji, N. Aldhafferi, and A. Alqahtani, "Ensemble-based support vector regression with gravitational search algorithm optimization for estimating magnetic relative cooling power of manganite refrigerant in magnetic refrigeration application," Journal of Superconductivity and Novel Magnetism, vol. 32, no. 7, pp. 2107-2118, 2019.

[29] A. Balogun, F. Rezaie, Q. B. Pham et al., "Spatial prediction of landslide susceptibility in western Serbia using hybrid support vector regression (SVR) with GWO, BAT and COA algorithms," Geoscience Frontiers, vol. 12, no. 3, p. 101104, 2021.

[30] T. O. Owolabi, "Extreme learning machine and swarm-based support vector regression methods for predicting crystal lattice parameters of pseudo-cubic/cubic perovskites," Journal of Applied Physics, vol. 127, no. 24, article 245107, 2020.

[31] S. M. I. Shamsah and T. O. Owolabi, "Modeling the maximum magnetic entropy change of doped manganite using a grid search-based extreme learning machine and hybrid gravitational search-based support vector regression," Crystals, vol. 10, no. 4, p. 310, 2020.

[32] M. Mitchell, “Genetic algorithms: an overview," Complex, John Wiley \& Sons, Inc, 1995.

[33] R. J. Thomas and S. Peethamparan, "Stepwise regression modeling for compressive strength of alkali-activated concrete," Construction and Building Materials, vol. 141, pp. 315-324, 2017.

[34] J. Xu, H. Zhang, L. Wang, W. Ye, W. Xu, and Z. Li, "QSPR analysis of infinite dilution activity coefficients of chlorinated organic compounds in water," Fluid Phase Equilibria, vol. 291, no. 2, pp. 111-116, 2010.

[35] M. J. Sharma and S. J. Yu, "Stepwise regression data envelopment analysis for variable reduction," Applied Mathematics and Computation, vol. 253, pp. 126-134, 2015.

[36] L. Jiao and H. Li, "QSPR studies on the aqueous solubility of PCDD/Fs by using artificial neural network combined with stepwise regression," Chemometrics and Intelligent Laboratory Systems, vol. 103, no. 2, pp. 90-95, 2010.
[37] M. Noryani, S. M. Sapuan, M. T. Mastura, M. Y. M. Zuhri, and E. S. Zainudin, "Material selection of natural fibre using a stepwise regression model with error analysis," Journal of Materials Research and Technology, vol. 8, no. 3, pp. 2865-2879, 2019.

[38] H. Ssegane, E. W. Tollner, Y. M. Mohamoud, T. C. Rasmussen, and J. F. Dowd, "Advances in variable selection methods I: causal selection methods versus stepwise regression and principal component analysis on data of known and unknown functional relationships," Journal of Hydrology, vol. 438-439, pp. 16-25, 2012.

[39] Y. Gu, Z. Bao, Y. Lin, Z. Qin, J. Lu, and H. Wang, "The porosity and permeability prediction methods for carbonate reservoirs with extremely limited logging data: stepwise regression vs. $\mathrm{N}$-way analysis of variance," Journal of Natural Gas Science and Engineering, vol. 42, pp. 99-119, 2017.

[40] G. Hu, Z. Xu, G. Wang, B. Zeng, Y. Liu, and Y. Lei, "Forecasting energy consumption of long-distance oil products pipeline based on improved fruit fly optimization algorithm and support vector regression," Energy, vol. 224, p. 120153, 2021.

[41] T. O. Owolabi, M. A. Abd Rahman, and A. Rahman, "Energy band gap modeling of doped bismuth ferrite multifunctional material using gravitational search algorithm optimized support vector regression," Crystals, vol. 11, no. 3, p. 246, 2021.

[42] A. A. Akinpelu, M. E. Ali, T. O. Owolabi et al., "A support vector regression model for the prediction of total polyaromatic hydrocarbons in soil: an artificial intelligent system for mapping environmental pollution," Neural Computing and Applications, vol. 32, no. 18, pp. 14899-14908, 2020.

[43] S. Durgam, A. Bhosale, V. Bhosale, R. Deshpande, and P. Sutar, "Support vector regression method for predicting temperatures of heat sources cooled by forced convection in a horizontal channel," Thermal Science and Engineering Progress, vol. 20, p. 100725, 2020.

[44] T. O. Owolabi, M. A. Suleiman, H. B. Adeyemo, K. O. Akande, J. Alhiyafi, and S. O. Olatunji, "Estimation of minimum ignition energy of explosive chemicals using gravitational search algorithm based support vector regression," Journal of Loss Prevention in the Process Industries, vol. 57, pp. 156-163, 2019.

[45] A. A. Motlagh, N. Shabakhty, and A. Kaveh, "Design optimization of jacket offshore platform considering fatigue damage using genetic algorithm," Ocean Engineering, vol. 227, article 108869, 2021.

[46] L. Gharsalli and Y. Guérin, "Mechanical sizing of a composite launcher structure by hybridizing a genetic algorithm with a local search method," Composites Part C: Open Access, vol. 5, article 100125, 2021.

[47] T. O. Owolabi, "Determination of the velocity of detonation of primary explosives using genetically optimized support vector regression," Prop, Explos, Pyrotech, vol. 44, no. 10, pp. 12821292, 2019.

[48] T. O. Owolabi, "Modeling the magnetocaloric effect of manganite using hybrid genetic and support vector regression algorithms," Physics Letters A, vol. 383, no. 15, pp. 1782-1790, 2019.

[49] Y. Wang and C. Wei, "Design optimization of office building envelope based on quantum genetic algorithm for energy conservation," Journal of Building Engineering, vol. 35, article 102048, 2021.

[50] A. A. Adewumi, M. Ismail, M. A. M. Ariffin et al., "Empirical modelling of the compressive strength of an alkaline activated 
natural pozzolan and limestone powder mortar," Ceram. - Silikaty, vol. 64, no. 4, pp. 407-417, 2020.

[51] M. Co, O. Fe, V. Verma, M. Kaur, and J. Marc, "Tailored structural, optical and magnetic properties of ternary nanohybrid," Ceramics International, vol. 45, no. 8, pp. 10865-10875, 2019.

[52] N. Amin, M. S. Ul Hasan, Z. Majeed et al., "Structural, electrical, optical and dielectric properties of yttrium substituted cadmium ferrites prepared by co-precipitation method," Ceramics International, vol. 46, no. 13, pp. 20798-20809, 2020.

[53] A. U. Rehman, N. A. Morley, N. Amin et al., "Controllable synthesis of $\mathrm{La} 3+$ doped $\mathrm{Zn} 0.5 \mathrm{Co} 0.25 \mathrm{Cu} 0.25 \mathrm{Fe} 2-\mathrm{xLaxO} 4$ ( $\mathrm{x}$ $=0.0,0.0125,0.025,0.0375,0.05)$ nano-ferrites by sol-gel auto-combustion route," Ceramics International, vol. 46, no. 18, pp. 29297-29308, 2020.

[54] K. Hussain, N. Amin, and M. I. Arshad, "Evaluation of structural, optical, dielectric, electrical, and magnetic properties of $\mathrm{Ce}^{3+}$ doped $\mathrm{Cu}_{0.5} \mathrm{Cd}_{0.25} \mathrm{Co}_{0.25} \mathrm{Fe}_{2-\mathrm{x}} \mathrm{O}_{4}$ spinel nano-ferrites," Ceramics International, vol. 47, no. 3, pp. 3401-3410, 2021.

[55] M. Dhiman, B. Chudasama, V. Kumar, K. B. Tikoo, and S. Singhal, "Augmenting the photocatalytic performance of cobalt ferrite via change in structural and optical properties with the introduction of different rare earth metal ions," Ceramics International, vol. 45, no. 3, pp. 3698-3709, 2019. 